\title{
Das Mammakarzinom im Medizinrecht
}

\section{Berg}

In der Gutachterstelle bei der Landesärztekammer Bayern wurden seit dem Jahr 2000 in 76 Fällen Beschwerden über Eingriffe an der weiblichen Brust vorgetragen. In 13 Fällen handelte es sich um kosmetische Eingriffe (Anerkennung des Vorwurfs in 2 Fällen), in 60 Fällen um Karzinome. Die Quote der anerkannten Fehler betrug 58,3\% ( $n=35)$. Das ist verglichen mit der Anerkennungsrate aller Beschwerden aus dem Bereich der Gynäkologie und Geburtshilfe von knapp 30\% außerordentlich viel.

Weitere Vorwürfe betrafen:

- fehlerhafte Operationen

- fehlerhafte Hormontherapie

- Unterlassung der Strahlentherapie

- Unterlassung der Röntgenkontrollen

- Extravasat (4)

- Beschwerden durch Markierungsclip

- Karzinomentstehung durch Östrogene

- Entstehung eines Sarkoms durch Nachbestrahlung

- Muskelriss durch Mammografie

Der Schwerpunkt der Vorwürfe liegt zweifellos beim übersehenen beziehungsweise zu spät erkannten Mamakarzinom. Die AG Medizinrecht der DGGG hat 2006 eine Leitlinie über „Das nicht erkannte Mammakarzinom“ publiziert [1], die letztmals im Oktober 2010 aktualisiert wurde. In kritischen Fällen wurde häufig zunächst ein eher als gutartig einzustufender Befund festgestellt und eine kurzfristige Kontrolle empfohlen. Die Patientin erscheint dann jedoch nicht. Monate später stellt sie selbst einen Knoten in der Brust fest. Der Arzt erklärt, er habe deutliche Warnhinweise gegeben und eine engmaschige Kontrolle empfohlen. Die Patientin sagt später, er habe alles bagatellisiert, bzw. nichts empfohlen. In der Patientenakte findet man keinen oder nur schwer zu deutende Hinweise auf eine erfolgte Aufklärung und Beratung. Oder die Patientin sagt, sie habe den Arzt immer wieder auf tastbare Knoten in ihrer Brust hingewiesen, ohne dass dieser die erforderlichen Konsequenzen gezogen hätte. Oder es wird eine Mammografie veranlasst, ein Befundbericht ist jedoch vom überweisenden Arzt nicht gesehen oder abgezeichnet worden und es wurde neun Monate später ein Karzinom entdeckt.

Abgesehen von dieser Leitlinie gibt es zwei S3-Leitlinien zur Brustkrebsdiagnostik bzw. -früherkennung [2], die den derzeitigen und forensisch bedeutsamen Standard der Diagnostik angeben und in denen das Vorgehen zur Krebserkennung beschrieben ist. Und schließlich gibt es bundesweit Programme zum Mammografie-Screening, die jeder Frau zwischen 50 und 70Jahren offenstehen.

Tab. 1

\begin{tabular}{|llcc|}
\hline $\begin{array}{l}\text { Vorwurf } \\
\text { Mammaplastik }\end{array}$ & $\mathbf{n}$ & anerkannt & abgelehnt \\
\hline $\begin{array}{l}\text { Mamma-Ca } \\
\text { zu spät erkannt* }\end{array}$ & 40 (offen: 2$)$ & 2 & 8 \\
\hline $\begin{array}{l}\text { fehlerhafte } \\
\text { Gewebsentnahme }\end{array}$ & 7 (offen: 2$)$ & 2 & $21(52,5 \%)$ \\
\hline \begin{tabular}{l} 
fehlerhafte Therapie \\
\hline
\end{tabular} & 11 & 3 & 8 \\
\hline
\end{tabular}

* In 11 Fällen richtete sich der Vorwurf gegen den Radiologen.
Kernpunkte der Diagnostik sind folgende:

- Grundsätzlich muss bei einer symptomatischen Brust (tastbarer Knoten oder Beschwerden jeglicher Art) so lange untersucht werden, bis ein Karzinom sicher ausgeschlossen ist.

- Ohne jeglichen Verdacht ist der Arzt allerdings nicht verpflichtet, bei einer Frau eine Mammografie durchzuführen oder zu empfehlen.

- Der Arzt darf sich auf das Ergebnis der Mammografie verlassen, wenn damit alle Zweifel ausgeräumt sind.

- Bei unklarem Befund sind weitere Untersuchungen (Mammasonografie, MRT) indiziert. Hilft auch das nicht weiter, sind invasive Verfahren (zum Beispiel Stanzbiopsie) zu erwägen.

- Empfohlene Kontrolltermine müssen klare zeitliche Vorgaben enthalten und dürfen sich nicht darauf beschränken, eine Wiedervorstellung zu empfehlen, „wenn sich der Befund nicht bessert".

- Dabei sollte mit der Patientin das Risiko erörtert werden, das sich bei Nichteinhaltung des Termins eventuell ergeben könnte. Derartige Ratschläge müssen sorgfältig dokumentiert werden.

- Es wird empfohlen, im Rahmen der horizontalen Arbeitsteilung mit dem Radiologen zu vereinbaren, dass auch er die Patientin über das Ergebnis einer Untersuchung informiert.

- Aus Gründen der haftungsrechtlichen Sicherheit wird auch empfohlen, eine Patientin, die nicht zum Gespräch über den Befund oder zum vereinbarten Kontrolltermin erscheint, schriftlich über das Ergebnis und eventuelle Empfehlungen zu Kontrolluntersuchungen zu informieren.

Der Dokumentation kommt eine erhebliche Bedeutung zu. Die unterlassene oder lückenhafte Dokumentation ist zwar keine eigene Anspruchsgrundlage für Schadensersatzansprüche, kann jedoch zu Beweiserleichterungen bis hin zur Beweislastumkehr führen. Es ist daher dringend zu empfehlen, Inhalte von Gesprächen und Beratungen wenigstens stichpunktartig zu dokumentieren und gegebenenfalls auch die Patientin an einen nicht eingehaltenen Folgetermin zu erinnern.

\section{Literatur}

1 AG Medizinrecht der DGGG: Das nicht erkannte Mammakarzinom. www.dggg.de/Leitlinien/ 2010

2 Albert US. Stufe-3-Leitlinie Brustkrebsfrüherkennung in Deutschland. Stufe 3-Leitlinie. Zuckschwerdt; 2008

\section{Bibliografie}

Dol http://dx.doi.org/10.1055/s-0031-1271520

Senologie 2011; 8: 105

(c) Georg Thieme Verlag KG Stuttgart · New York · ISSN 1611-6453

Korrespondenzadresse

Prof. em Dr. Dietrich Berg

Schwaiger Str. 33

92224 Amberg

dberg@asamnet.de 